\title{
Current and future prospects for optoelectronic retinal prostheses
}

J Dowling

\section{Dowling}

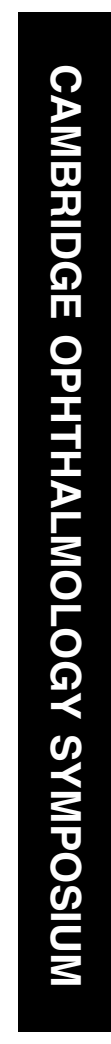

photoreceptors (although recent drug therapies for AMD, such as Lucentis, might shift the focus of current treatments to RP). In 1956, the Australian researcher Tassicker ${ }^{3}$ was the first to patent a method of implanting a light-sensitive selenium photodiode behind a blind person's retina to restore intermittent light sensation. Influenced by the success of the cochlear implant in stimulating secondary neurons to transfer sensory information, early retinal prosthesis researchers examined whether retinocortical connections survived in profoundly blind people. ${ }^{4}$ Results from post-mortem examinations of profoundly blind people showed $80 \%$ of the inner nuclear layer and approximately $30 \%$ of the ganglion cell layer to be spared. ${ }^{5}$

For a retinal visual prosthesis to work, there must be some functional retinal ganglion cells (RGCs), and therefore these systems are not capable of treating conditions such as glaucoma and diabetic retinopathy. Prosthesis systems that target the visual cortex, optic nerve, ${ }^{6}$ or the dorsal lateral geniculate nucleus of the thalamus ${ }^{7}$ may provide solutions for these conditions.

There are considerable challenges to the development of a successful retinal prosthesis. Significant issues include limitations to the number of electrodes, biocompatibility, encapsulation, electrode degradation, power requirements, signal and image processing, interference with residual vision, functional evaluation, and training. Despite these issues, currently, more than 20 different groups (for a recent list see Rizzo et $^{\mathrm{a}} \mathrm{l}^{8}$ ) are researching the development of intraocular (subretinal and epiretinal, and suprachoroidal) and extraocular (trans- and episcleral) retinal prostheses.

\section{Optoelectronic approaches}

A focus of this review is on optoelectronic prostheses, or systems that involve the direct conversion of energy from light to electricity
CSIRO ICT Centre, The Australian e-Health Research Centre BioMedIA, Level 7, Royal Brisbane and Women's Hospital, Herston, Queensland, Australia

Correspondence: J Dowling, CSIRO ICT Centre,

The Australian e-Health Research Centre BioMedIA, Level 7, UQ CCR Building 71/918,

Royal Brisbane and Women's Hospital, Herston, Queensland QLD 4029, Australia

Tel: + 6173024 1634; Fax: + 61732533690 E-mail: Jason.Dowling@ csiro.au

Received: 22 September 2008

Accepted: 30 September 2008

Published online: 19

December 2008

Presented at the 38th

Cambridge

Ophthalmological

Symposium 
(typically from photodiode arrays). Generally, the optoelectronic approaches have concentrated on subretinal stimulation, where the implant is located behind the photoreceptor layer and in front of the retinal pigment epithelium. The advantage of this location is that existing information-reduction functions in the inner nuclear layer (amacrine, bipolar, and horizontal cell nuclei) may still be utilised (unlike epiretinal approaches, which are more likely to stimulate the retinal ganglion and amacrine cells directly).

In this section, four systems based primarily on optoelectronic power transfer and at various stages of development are discussed. The first company, Optobionics Corporation, founded in 1990, developed an 'artificial silicon retina' (ASR) consisting of 5000 microelectrode-tipped passive microphotodiodes (powered purely from ambient light). After a number of successful in vitro and in vivo animal trials, Optobionics received approval from the US Food and Drug Administration (FDA) to commence safety and feasibility trials in six patients, and a 2-mm diameter passive multiphotodiode array (MPDA) was implanted in the right eye of six legally blind patients with RP. All patients reported improvements in visual function; however, these were unlikely to have been solely from retinal electrical stimulation, as improvement did not occur immediately but over several weeks and occurred in both stimulated and unstimulated areas of the visual field. Therefore, the ASR appeared to have triggered neurotrophic-type mechanisms in the retina. ${ }^{9}$ An involuntary petition to liquidate was filed against Optobionics in 2007.

A Southern German subretinal prosthesis team led by the University Eye Hospital in Tubingen was formed in
1995. Significant early research involved both in vitro and in vivo experiments aimed at determining threshold parameters and surgical techniques. ${ }^{10}$ In 2003, the company Retina Implant was formed from this group. As natural photoreceptors are much more efficient than photodiodes, the group chose to install an additional set of photodiodes that are irradiated by infrared light and generate electrical power to supply the amplification circuits in the retinal implant, ${ }^{11}$ before developing an active power source (where the photodiodes are modulating current delivered from an external source) (Figure 1). In 2006, an active hybrid 1500 microphotodiode array (MPDA) was implanted into the subretinal space of six patients for 30 days (although one patient later refused to have it removed). Two of these patients reported phosphenes through the MPDA, although only when larger areas of the array were illuminated (not for a single photodiode/electrode). In addition to the microphotodiodes, 16 direct stimulation electrodes were also included in the device, which were found to elicit phosphenes in four subjects. Some complications were reported, including mild macular oedema. Perception of phosphenes appeared to rely on stimulation of a three- or four-electrode neighbourhood, ${ }^{12}$ which may be due to the interference between on and off receptors during simultaneous stimulation. Retina Implant intends to release a commercial product by the beginning of 2010 .

An alternate optoelectronic approach has been developed by Palanker et $a l^{13}$ at Stanford University. Their proposed system is similar in design to that of Optobionics and the Retina Implant system. The significant difference is the use of goggle-mounted infrared projection system to activate implanted

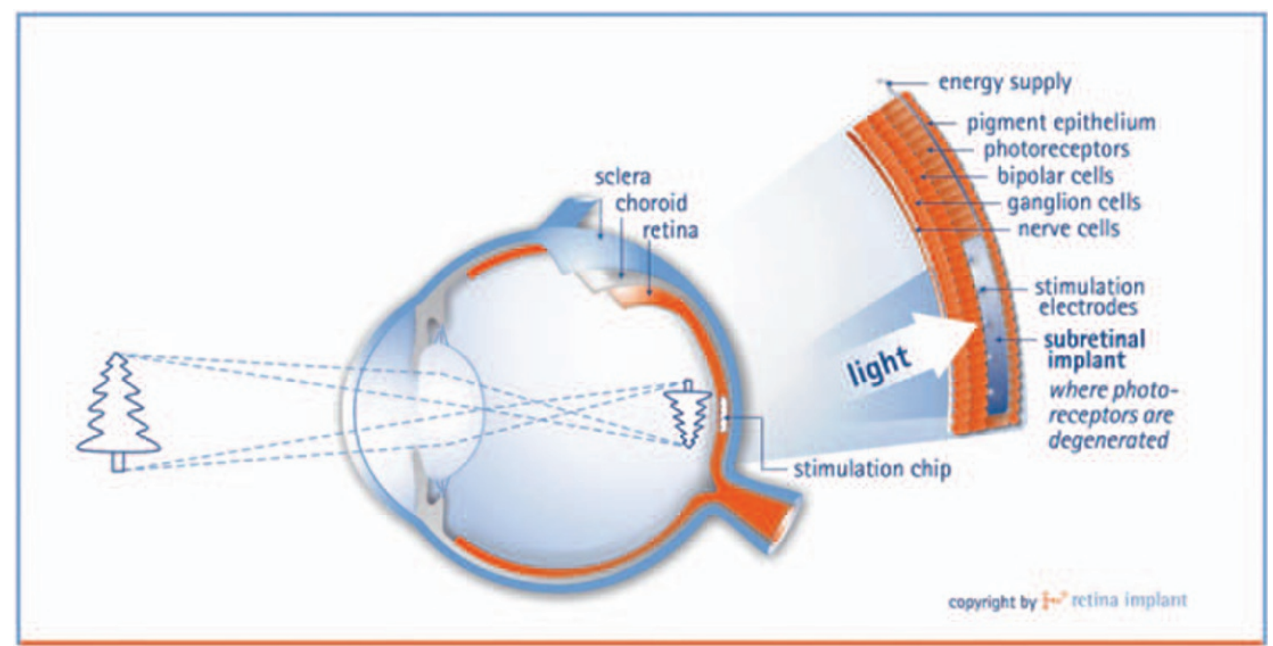

Figure 1 Diagram of the active photodiode optoelectronic subretinal prosthesis developed by Retina Implant ${ }^{12}$ (reprinted with permission from Retina Implant). 
photodiodes (Figure 2). Owing to the transparency of these goggles to normal light, the system could be used to supplement remaining vision with phosphene perception. The system can be used with both subretinal and epiretinal implants. A real-time tracking system is being developed that monitors landmark points on the implanted chip, allowing the image projected from the LCD display to be adjusted to the current position of the chip. Although the implant covers only $10^{\circ}$ of the visual field, the projected infrared image corresponds to a larger field of view $\left(30^{\circ}\right)$ than most retinal prostheses, and is

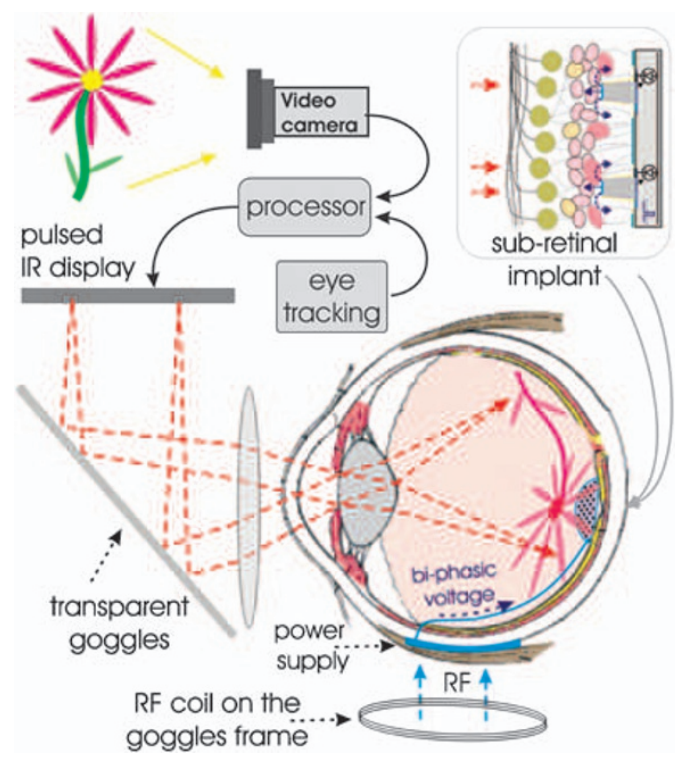

Figure 2 Design of the optoelectronic system under development by Palanker et al at Stanford University. Captured images are sent through a goggle-mounted infrared projection system to activate implanted photodiodes (reprinted with permission from IOP Publishing ${ }^{14}$ ). accessible by eye movements. The system also allows location-dependent image processing. ${ }^{15}$ More recently, ${ }^{14}$ the use of a laser diode for enhanced brightness of the image-projection system has been described. An additional benefit of the photovoltaic version of the system is that pixels operate independently and they can be placed in different locations within the subretinal space. Charge injection has now been maximised by applying a common bias voltage provided from a gogglemounted radiofrequency coil. To ensure that electrodes are close to target cells, Palanker et al ${ }^{13}$ have also developed techniques allowing the retina to migrate very closely to the implant.

Recently, a novel photostimulation approach has been proposed by a group from Imperial College, London, that involves virally re-engineering RGCs and/or bipolar cells to become light sensitive. ${ }^{16}$ These cells could then be stimulated to fire action potentials by the use of a headmounted gallium nitride LED array (a similar design to modern virtual reality headsets) (Figure 3). A unique feature of this approach is the potential to inhibit as well as excite action potentials. Other advantages include minimal damaging surgery, the lack of an implanted cable or power supply, and the ability to target individual receptive fields and cells. ${ }^{17}$ Although in vivo animal studies are planned to commence in the next year, human testing is still some years away. The system will only be useful for people without light perception, as any remaining photoreceptors will be quickly bleached by the high-intensity light.

Alternate preliminary biochemical approaches to retinal prosthetics have also been proposed by Greenbaum et $a l^{18}$ (where light-absorbing pigments from spinach are added to ganglion or bipolar cells) and Chambers et $a l^{19}$ (who suggest a ganglion-cell stimulation method based on semisynthetic light-activated ion channels).

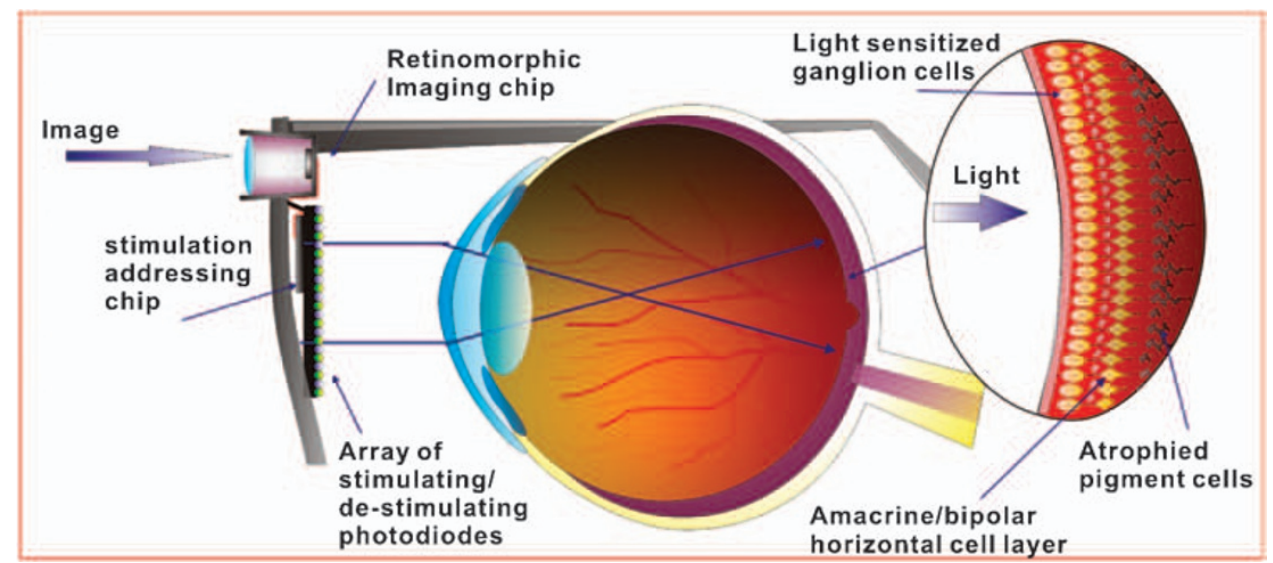

Figure 3 Conceptual overview of the optoelectronic retinal prosthesis in development by Degenaar and co-workers ${ }^{16}$ at Imperial College. A head-mounted LED array stimulates light-sensitive ganglion cells. 


\section{External power-based approaches to retinal prostheses}

An alternative to optoelectronic prostheses is the use of implanted multielectrode arrays (MEAs) and

transcutaneous telemetry to transfer data and power. An advantage with this approach is that image capture and processing can take place in external, commercially available devices (such as a head-mounted camera linked to a pocket PC) that can be easily upgraded or replaced.

Second Sight, based in California, is currently the leading visual prosthetics research group and utilises an epiretinal MEA. Seminal research by one of the Second Sight collaborators, Humayun et $a l^{20}$, demonstrated successful phosphene perception from local electrical stimulation of the retina in 14 patients in 1995. Further study has resulted in a functioning system consisting of a glass-mounted camera and an external image-processing unit connected through a telemetric inductive link to the intraocular unit (containing a power source, signal transceiver, stimulation unit, and electrode array). ${ }^{21}$ Between 2002 and 2004, Second Sight conducted acute and chronic testing of a 16-electrode device (the Argus 16). Six subjects with bare light perception secondary to RP were enrolled in this trial and were able to use spatial information from the stimulator to detect motion (such as a bar moving in front of the camera) and distinguish common household objects. ${ }^{22}$ Successful in vivo animal studies of the Argus II implant, consisting of 60 independently controllable electrodes, have been reported, and in 2007, the US FDA approved the device for clinical study in humans. $^{23}$

Intelligent Medical Implants have also developed an epiretinal 'learning retinal implant system', similar in design to the Second Sight model. The main feature of this system is an 'adaptive retina encoder' developed to assist with the adjustment of stimulation parameters for individual patients. Acute testing has been carried out on 20 subjects with RP, 19 of whom reported small phosphenes with different shapes and in a variety of colours. ${ }^{24}$ Chronic testing of the learning retinal implant has also been ongoing since late 2005, although results are unavailable.

The Boston Retinal Implant Project that conducted an important early study on an epiretinal prosthesis, however, later changed their focus to developing a 15-electrode subretinal implant, with most of the device located outside the eye. The use of iridium oxide (rather than platinum) allows smaller electrodes, which in theory are capable of delivering higher levels of charge. Acute studies have been performed successfully with five patients with RP and one sighted patient. ${ }^{25,26}$

A second German epiretinal prosthesis group, Epiret $\mathrm{GmbH}$, has also recently successfully implanted a 25-electrode array (EPIRET3) into six patients over a 4-week period. ${ }^{23}$ The implant was tolerated in five eyes with moderate post-operative inflammation, and the position of the implants remained stable until removal. ${ }^{27}$

Two Australian research groups are involved in the development of retinal prostheses. The Australian Vision Prosthesis Group (AVPG) has designed an epiretinal prosthesis with a similar design to Second Sight, in which power and data are sent to an intraocular implant through a transcutaneous inductive link. ${ }^{28}$ A feature of the implant is the unique hexagonal arrangement of electrodes that can assist the localisation of current spread through the neural retina ${ }^{29}$ (Figure 4). The AVPG has conducted research into simulation (see below), both internal and external hardware, and animal model experiments. ${ }^{30}$ The group aims to commence acute human testing of a suprachoroidal electrode placement within the next 2 years (followed by chronic testing).

The second Australian group, the Australian Bionic Eye Foundation, aims to adapt MEA technology from Cochlear Ltd to stimulate the peripheral retina in patients with RP (aiming to increase the peripheral visual field to assist with orientation and mobility). In an extraocular approach, the group has adapted an 18-electrode brain stem implant to be attached outside the sclera. The advantages of this location are that the macula is not at risk of further damage and intraocular surgery is avoided. An acute study of 17 patients with RP and 15
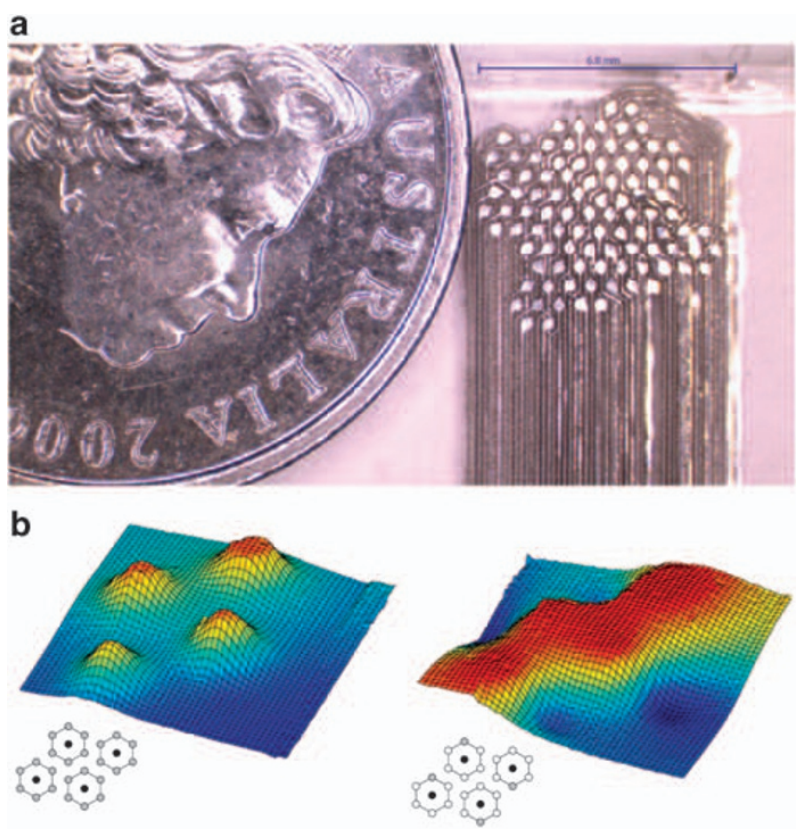

Figure 4 (a) Photograph of the AVPG microelectrode array. (b) A comparison of saline bath recording using the AVPG hexagonal guard technique (left) compared with other single guard methods (right). A significant improvement in current localisation can be observed. 
normally sighted patients has been conducted using contact lens electrodes, and minimum current thresholds for phosphene perception have been identified. ${ }^{31}$ A potential disadvantage of the group's approach is the increased distance between electrodes and target neurons, resulting in a lower resolution and high charge required to reach activation thresholds. ${ }^{32}$

A group from Osaka University, Japan, has also been working towards the development of an extraocular retinal prosthesis, with the anodic-stimulating electrode located in the suprachoroid layer and the cathode located in the vitreous body. The implant was found to generate focal excitation in RGCs in normal animals and in those with degenerated photoreceptors, and thresholds for a safe current have been evaluated. ${ }^{33}$ Acute experiments using transcorneal and transscleral simulation in 14 normally sighted and two RP patient volunteers have been performed to obtain threshold parameters. All volunteers reported a round-shaped phosphene in response to transscleral stimulation, and phosphene brightness could be controlled with changes in pulse frequency. ${ }^{34}$ As with the Australian Bionic Eye Foundation device, the separation of neural cells from electrodes may cause problems due to overheating, crosstalk, and electrochemical erosion, ${ }^{13}$ which may restrict the potential visual acuity of the suprachoroidaltransretinal approach. However, the study by Wong et $a l^{35}$ in cats using suprachoroidal electrode placement has demonstrated the generation of localised phosphene responses with appropriate electrode configurations.

\section{Image-processing and simulation research}

Most of the systems described require an external camera for image acquisition. Apart from the real-time subsampling and conversion of these images to electrode-stimulation patterns, adaptive image processing will probably be required to adjust the resolution, contrast, and brightness of captured images. Non-uniform stimulation thresholds may be required due to the uneven thickness or wavy structure of a diseased retina, ${ }^{13}$ and the possibility of continued remodelling of the retina post-implantation could result in spatial corruption and cryptic synapse formation. ${ }^{36}$ Epiretinal implants stimulate the RGC layer directly and these cells expect the output from the pre-processed and filtered visual information (from bipolar and amacrine cells), not a camera image. ${ }^{17}$ Some of the prosthesis systems discussed aim at replicating these functions (such as the learning retina system from Intelligent Medical Implants and the Stanford University system).

A number of simulation studies have been conducted to determine requirements and limitations of visual prosthesis systems. These studies have involved either printed or PC monitor-based static images, or headmounted simulation devices. Frequently cited simulator research by $\mathrm{Cha}$ et $a l^{37}$ evaluated the effects of simulated phosphene density on walking speed and obstacle contacts, reporting that a successful visual prosthesis requires a $25 \times 25$ array of phosphenes with a $30^{\circ}$ field of view. A recent study has investigated frame rate and phosphene resolution in the development of adaptive image processing. ${ }^{38}$

A range of approaches have been used to simulate the phosphenes reported by patients, with uniform Gaussian intensity used by most groups (for example, Dowling et $a l^{38}$ and Dagnelie $\left.e t a l^{39}\right)$. An advanced image-analysis method for constructing phosphenes was presented in Hallum et $a l^{40}$ that could be useful in enhancing the effectiveness of individual patient parameter tuning Investigation of salient regions in static simulation images has been investigated by Boyle et $a l^{41}$ who reported benefits in using enhanced zoom in areas of interest. Hayes et $a l^{42}$ investigated the effect of various parameters (such as percentages of missing phosphenes from an array and phosphene size) on object recognition concluding that the number of phosphenes was the most important parameter. Phosphene face-recognition research $^{43}$ has been found to be acceptable with a $10 \times 10$ array of phosphenes, and the authors noted significant subject-learning effects during trials. In addition, a number of papers have attempted to determine reading requirements. ${ }^{44-48}$ There has been considerable research in image-processing algorithms to assist the visually impaired, which could be integrated into visual prosthesis systems.

As most systems use a head-mounted camera, increased head movements are required to direct gaze and to scan visual scenes. The use of increased head movements by cortical implant recipients was noted by Dobelle. ${ }^{2}$ Head scanning has been investigated by Chen et $a l^{49}$ who suggest that scanning can be used to increase spatial frequency through temporal integration.

\section{Future prospects}

The prospects for retinal prostheses in restoring some visual substitution to those blinded by RP or AMD in general appear promising. As a number of companies are competing to produce a commercial visual prosthesis, bias in reported findings has been noted. ${ }^{23}$ Three companies aim to release commercial devices before the end of 2010: Second Sight, Intelligent Medical Implants, and Retina Implant.

In the short term, microelectrode array systems (particularly Second Sight) appear to hold an advantage. The main limitation with the Second Sight system appears to be spatial resolution, which could be 
improved with an increase of stimulating electrodes. However, this increase is constrained by increased safety concerns, the potential for cross-talk, and increased power requirements. ${ }^{50}$ Hermetic encapsulation is also a concern for all intraocular devices.

Three epiretinal companies have ongoing clinical studies with chronic implantation: Second Sight, Intelligent Medical Implants, and EpiRet Gmbh. Passive MPDA devices (as used by Optobionics) appear to have failed. Active MPDAs show more promise, and Retina Implant is currently the only subretinal prosthesis to have chronic clinical trials. Three different groups (Boston, Osaka University, and the Australian Bionic Eye Foundation) have completed acute clinical studies.

The biochemical retinal prosthesis approaches (such as Imperial College) appear to be at least 5 years away from commercialisation.

In summary, the restoration of some vision using these devices will be a great achievement. However, none of these retinal prosthesis systems will restore anything resembling normal vision in the near future. Current mobility constraints include the narrow field of view $\left(\sim 10^{\circ}\right)$ and the need for head-movement scanning (due to the head-mounted camera). As the field matures, interaction with orientation and mobility staff and the development of pre- and post-operative training programmes and functional evaluation measures will become increasingly important.

\section{Acknowledgements}

I gratefully acknowledge discussions and comments on this paper by the following researchers: Vivek Chowdhury (Australian Bionic Eye Foundation), Gislin Dagnelie (Johns Hopkins University, USA), Patrick Degenaar (Imperial College, UK), Takashi Fujikado (Osaka University, Japan), Nigel Lovell (AVPG, University of New South Wales, Australia), Anthony Maeder (University of Western Sydney, Australia), Peter Meijer (Phillips, The Netherlands), Daniel Palanker (Stanford University, USA), Gregg Suaning (AVPG, University of New South Wales, Australia), and Walter Wrobel (Retina Implant AG, Germany).

\section{References}

1 Brindley GS, Lewin WS. The sensations produced by electrical stimulation of the visual cortex. J Physiol 1968; 196(2): 479-493.

2 Dobelle W. Artificial vision for the blind by connecting a television camera to the brain. ASAIO J 2000; 46(1): 3-9.

3 Tassicker GE. Preliminary report on a retinal stimulator. $\mathrm{Br}$ J Physiol Opt 1956; 13(2): 102-105.

4 Dagnelie G. Psychophysical evaluation for visual prosthesis. Annu Rev Biomed Eng 2008; 10: 339-368.
5 Stone JL, Barlow WE, Humayun MS, de Juan Jr E, Milam $\mathrm{AH}$. Morphometric analysis of macular photoreceptors and ganglion cells in retinas with retinitis pigmentosa. Arch Ophthalmol 1992; 110: 1634-1639.

6 Brelén ME, De Potter P, Gersdorff M, Cosnard G, Veraart C, Delbeke J. Intraorbital implantation of a stimulating electrode for an optic nerve visual prosthesis. Case report. J Neurosurg 2006; 104(4): 593-597.

7 Pezaris JS, Reid RC. Demonstration of artificial visual percepts generated through thalamic microstimulation. Proc Natl Acad Sci USA 2007; 104(18): 7670-7675.

8 Rizzo JF, Snebold L, Kenney M. Development of a visual prosthesis. In: Tombran-Tink J, Barnstable C, Rizzo JF (eds). Visual Prosthesis and Ophthalmic Devices: New Hope in Sight. Humana Press Inc.: Totawa, New Jersey, 2007, pp 71-93.

9 Chow AY, Chow VY. Subretinal artificial silicon retina microchip implantation in retinitis pigmentosa. In: Tombran-Tink J, Barnstable C, Rizzo JF (eds). Visual Prosthesis and Ophthalmic Devices: New Hope in Sight. Humana Press Inc.: Totawa, New Jersey, 2007, pp 37-54.

10 Zrenner E. The subretinal implant: can microphotodiode arrays replace degenerated retinal photoreceptors to restore vision? Ophthalmologica 2002; 216(Suppl 1): 8-20.

11 Schubert MB, Hierzenberger A, Lehner HJ, Werner JH. Optimizing photodiode arrays for the use as retinal implants. Sens Actuators A Phys 1999; 74(1-3): 193-197.

12 Zrenner E. Restoring neuroretinal function by subretinal microphotodiode arrays. Presentation at ARVO, Fort Lauderdale, USA 2007.

13 Palanker D, Vankov A, Huie P, Baccus S. Design of a highresolution optoelectronic retinal prosthesis. J Neural Eng 2005; 2(1): S105-S120.

14 Loudin JD, Simanovskii DM, Vijayraghavan K, Sramek CK, Butterwick AF, Huie P et al. Optoelectronic retinal prosthesis: system design and performance. J Neural Eng 2007; 4(1): S72-S84.

15 Asher A, Segal WA, Baccus SA, Yaroslavsky LP, Palanker DV. Image processing for a high-resolution optoelectronic retinal prosthesis. IEEE Trans Biomed Eng 2007; 54(6 Part 1): 993-1004.

16 Poher V, Grossman N, Kennedy GT, Nikolic K, Zhang HX, Gong $\mathrm{Z}$ et al. Micro-LED arrays: a tool for two-dimensional neuron stimulation. J Phys D Appl Phys 2008; 41: 94014-94022.

17 Nikolic K, Grossman N, Yan H, Drakakis E, Toumazou C, Degenaar P. A non-invasive retinal prosthesis - testing the concept. Proceedings of the 29th Annual Conference of the IEEE EMBS 2007; 2007: 6365-6368.

18 Greenbaum E, Humayun MS, Kuritz T, Lee JW, Sanders CA, Bruce B et al. Nanoscale photosynthesis, the photophysics of neural cells, and artificial sight. Proceedings of the IEEEEMBS Special Topic Conference on Molecular, Cellular and Tissue Engineering 2002; 2002: 83-85.

19 Chambers JJ, Banghart MR, Trauner D, Kramer RH. Lightinduced depolarization of neurons using a modified shaker $\mathrm{K}+$ channel and a molecular photoswitch. J Neurophysiol 2006; 96: 2792-2796.

20 Humayun MS, Sato Y, Propst R, de Juan Jr E. Can potentials from the visual cortex be elicited electronically despite severe retinal degeneration and a markedly reduced electroretinogram? Ger J Ophthalmol 1995; 4(1): 57-64

21 Liu W, Sivaprakasam M, Wang G, Zhou M, Weiland JD, Humayun MS. Challenges in realizing a chronic highresolution retinal prosthesis. In: Humayun MS, Chader G, 
Weiland JD (eds). Artificial Sight: Basic Research, Biomedical Engineering, and Clinical Advances. Springer-Verlag: New York, 2007, pp 129-149.

22 Weiland JD, Humayun MS. Visual prosthesis. Proceedings of the IEEE 2008; 96(7): 1076-1084.

23 Sommerhalder J. ARVO annual meeting 2008: visual prostheses research. Exp Rev Ophthalmol 2008; 3(4): 389-391.

24 Hornig R, Zehnder T, Velikay-Parel M, Laube T, Feucht M, Richard G. The IMI retinal implant system. In: Humayun MS, Chader G, Weiland JD (eds). Artificial Sight: Basic Research, Biomedical Engineering, and Clinical Advances. Springer-Verlag: New York, 2007, pp 111-128.

25 Rizzo J, Wyatt J, Loewenstein J, Kelly S, Shire D. Perceptual efficacy of electrical stimulation of human retina with a microelectrode array during short-term surgical trials. Invest Ophthalmol Vis Sci 2003; 44(12): 5362-5369.

26 Rizzo J, Wyatt J, Loewenstein J, Kelly S, Shire D. Methods and perceptual thresholds for short-term electrical stimulation of human retina with microelectrode arrays. Invest Ophthalmol Vis Sci 2003; 44(12): 5355-5361.

27 Roessler G. Clinical results for the EPIRET3 clinical trial-implantation and explanation of a wireless system for retinal stimulation. The Eye and The Chip - World Congress on Artificial Vision. Detroit, USA 2008.

28 Wong YT, Dommel NB, Lehmann T, Preston PJ, Lovell NH, Suaning GJ. Retinal neurostimulator for a multi-focal vision prosthesis. IEEE Trans Neural Syst Rehabil Eng 2007; 15(3): 425-434.

29 Hallum LE, Suaning GJ, Lovell NH. Contribution to the theory of prosthetic vision. ASAIO J 2004; 50(4): 392-396.

30 Kerdraon YA, Downie JA, Suaning GJ, Capon MR, Coroneo MT, Lovell NH. Development and surgical implantation of a vision prosthesis model into the ovine eye. Clin Exp Ophthalmol 2002; 30(1): 36-40.

31 Chowdhury V, Huang QH, Coroneo MT. Extraocular retinal prosthesis. The Eye and The Chip-World Congress on Artificial Vision. Detroit 2008.

32 Siu T, Morley J. Implantation of episcleral electrodes via anterior orbitotomy for stimulation of the retina with induced photoreceptor degeneration: an in vivo feasibility study on a conceptual visual prosthesis. Acta Neurochirurgica 2008; 150(5): 477-485.

33 Nakauchi K, Fujikado T, Kanda H, Kusaka S, Ozawa M, Sakaguchi $\mathrm{H}$ et al. Threshold suprachoroidal-transretinal stimulation current resulting in retinal damage in rabbits. J Neural Eng 2007; 4: S50-S57.

34 Fujikado T, Morimoto T, Kanda H, Kusaka S, Nakauchi K, Ozawa $\mathrm{M}$ et al. Evaluation of phosphenes elicited by extraocular stimulation in normals and by suprachoroidaltransretinal stimulation in patients with retinitis pigmentosa. Graefes Arch Clin Exp Ophthalmol 2007; 245(10): 1411-1419.

35 Wong YT, Chen SC, Kerdraon YK, Allen PJ, McCombe MF, Morley JW et al. Efficacy of supra-choroidal, bipolar, electrical stimulation in a vision prosthesis. Proceedings of the 30th Annual International Conference of the IEEE Engineering in Medicine and Biology Society. Vancouver, Canada 2008.

36 Marc RE, Jones BW, Watt CB, Strettoi E. Neural remodeling in retinal degeneration. Progr Retin Eye Res 2003; 22(5): 607-655.

37 Cha K, Horch K, Normann R. Mobility performance with a pixelised vision system. Vision Res 1992; 32(7): 1367-1372.

38 Dowling J, Maeder A, Boles W. Visual prostheses for the blind: a framework for information presentation. In: Billingsley J, Bradbeer R (eds). Mechatronics and Machine Vision in Practice. Springer: Berlin, 2008, pp 275-287.

39 Dagnelie G, Keane P, Narla V, Yang L, Weiland J, Humayun M. Real and virtual mobility performance in simulated prosthetic vision. J Neural Eng 2007; 4(1): S92-S101.

40 Hallum LE, Cloherty SL, Lovell NH. Image analysis for microelectronic retinal prosthesis. IEEE Trans Biomed Eng 2008; 55(1): 344-346.

41 Boyle JR, Maeder AJ, Boles WW. Region-of-interest processing for electronic visual prostheses. J Electron Imaging 2008; 17(1): 013002-013013.

42 Hayes JS, Yin VT, Piyathaisere D, Weiland JD, Humayun MS, Dagnelie G. Visually guided performance of simple tasks using simulated prosthetic vision. Artif Organs 2003; 27(11): 1016-1028.

43 Thompson R, Barnett G, Humayun M, Dagnelie G. Facial recognition using simulated prosthetic pixelized vision. Invest Ophthalmol Vision Sci 2003; 44(11): 5035-5042.

44 Cha K, Horch K, Norman R. Reading speed with a pixelized vision system. J Opt Soc Am A 1992; 9(5): 673-677.

45 Fu L, Cai S, Zhang H, Hu G, Zhang X. Psychophysics of reading with a limited number of pixels: towards the rehabilitation of reading ability with visual prosthesis. Vision Res 2006; 46(8-9): 1292-1301.

46 Dagnelie G, Barnett D, Humayun MS, Thompson Jr RW. Paragraph text reading using a pixelized prosthetic vision simulator: parameter dependence and task learning in freeviewing conditions. Invest Ophthalmol Vis Sci 2006; 47(3): 1241-1250.

47 Sommerhalder J, Oueghlani E, Bagnoud M, Leonards U, Safran AB, Pelizzone M. Simulation of artificial vision: I. Eccentric reading of isolated words, and perceptual learning. Vision Res 2003; 43(3): 269-283.

48 Sommerhalder J, Rappaz B, de Haller R, Fornos AP, Safran $A B$, Pelizzone M. Simulation of artificial vision: II. Eccentric reading of full-page text and the learning of this task. Vision Res 2004; 44(14): 1693-1706.

49 Chen SC, Hallum LE, Suaning GJ, Lovell NH. A quantitative analysis of head movement behaviour during visual acuity assessment under prosthetic vision simulation. J Neural Eng 2007; 4(1): S108-S123.

50 Winter JO, Cogan SF, Rizzo III JF. Retinal prostheses: current challenges and future outlook. J Biomater Sci 2007; 18(8): 1031-1055. 